\title{
$N$-SPECIES COMPETITION IN A PERIODIC CHEMOSTAT
}

\author{
Gail S.K. Wolkowicz† And XiaO-Qiang ZhaO $\ddagger$ \\ Department of Mathematics and Statistics, McMaster University \\ Hamilton, Ontario, Canada L8S 4K1
}

(Submitted by: Glenn Webb)

\begin{abstract}
A threshold result on the global dynamics of the scalar asymptotically periodic Kolmogorov equation is proved and then applied to models of singlespecies growth and $n$-species competition in a periodically operated chemostat. The operating parameters and the species-specific response functions can be periodic functions of time. Species-specific removal rates are also permitted. Sufficient conditions ensure uniform persistence of all of the species and guarantee that the full system admits at least one positive, periodic solution. In the special case when the species-specific removal rates are all equal to the dilution rate, the single-species growth model has a threshold between global extinction and uniform persistence, in the form of a positive, periodic coexistence state. Improved results in the case of 3 -species competition are also given, including an example illustrating competitionmediated coexistence of three species.
\end{abstract}

1. Introduction. The chemostat is an important laboratory apparatus used for the continuous culture of microorganisms. In ecology it is often viewed as a model of a simple lake system, of the wastewater treatment process, or of biological waste decomposition. It is an excellent experimental venue in which to study the effect of simple microbial interactions, including exploitative competition.

Various mathematical models have been developed and analyzed extensively by many different investigators (see, for example, the recent monograph by Smith and Waltman ([13]) and the references therein). Mathematical models of the chemostat are among the few predictive models in microbial ecology. The models of exploitative competition in a well-stirred chemostat operated under constant input and dilution, with competition for a nonreproducing substrate, predict competitive exclusion. That is, they predict that at most one competitor population avoids extinction (see, e.g.,

Accepted for publication February 1997.

$\dagger$ Research supported in part by the NSERC of Canada.

$\ddagger$ On leave from the Institute of Applied Mathematics, Academia Sinica, Beijing 100080, China. Research supported in part by the NSF of China.

AMS Subject Classifications: 34C25, 34D05, 34D45, 92D25, 92D40. 
$[2,13,15,16])$. However, the coexistence of competing populations is obvious in nature, and so in order to explain this, it seems necessary to relax at least one of the assumptions in the above models. One natural approach is to introduce periodic coefficients to represent, for example, daily or seasonal variations in the environment.

There has been some research on models of the chemostat involving either periodic nutrient input or periodic dilution rates (see, e.g., [1, 4-5, 7-10, 13, 14, 17]). As well, the periodic gradostat has been considered (see Smith $[11-12])$.

In most of the previous analytical studies of the periodic chemostat, the powerful theory of monotone dynamical systems was applied to limiting systems obtained using certain conservation principles. However, the theory of monotone dynamical systems can only be applied in this context to study the competition between at most two species. Also, in order to apply a conservation law to obtain the limiting systems, it is necessary to assume that all of the removal rates are equal, thus ignoring all the species-specific death rates and only considering the dilution rate.

Using a bifurcation theory approach, Lenas and Pavlou ([8-9]) used the numerical package AUTO to study two-species and three-species competition models in a periodically operated chemostat. Their results indicate that for certain parameter ranges, their model admits quasi-periodic and chaotic coexistence.

Our aim in this paper is to present a general framework to study, analytically, models of $n$-species competition in a periodically operated chemostat. Nutrient input, dilution and species-specific removal rates are all permitted to be periodic (but of commensurate period). As well, each species-specific nutrient uptake function is assumed to be a monotone increasing function of the substrate concentration, but can be periodic as a function of time (but again of commensurate period). Differential species-specific removal rates are also permitted. We apply the theory of asymptotically periodic semiflows (see Zhao [21]) and the comparison method (see Coppel [3]) to determine criteria that guarantee the existence of at least one positive periodic solution for the full system and the uniform persistence of all of the interacting species. For 3 -species competition, in the case that all the species-specific removal rates are assumed to be equal to the dilution rate, under additional assumptions on the form of the attractors in the lower-dimensional subsystems, abstract persistence theory for periodic semiflows (see Zhao [20]) is used to obtain similar results under criteria that are more easily satisfied.

This paper is organized as follows. In Section 2, a threshold-type result on the global dynamics of the scalar asymptotically periodic Kolmogorov equation is first proved by using asymptotically periodic semiflow theory 
(Theorem 2.1). Then single-species growth in the periodically operated chemostat is considered. In the case that the species-specific removal rate is permitted to be different than the dilution rate, Theorem 2.1 and the comparison method are used to obtain sufficient conditions that guarantee the existence of at least one positive periodic solution and ensures that the species is uniformly persistent (that is, regardless of the initial concentration of the species, provided that it is positive, the concentration of the species remains uniformly bounded away from zero). On the other hand, when the species-specific removal rate is assumed to be equal to the dilution rate, a threshold-type result for the uniform persistence versus global extinction of the species is obtained (Corollary 2.3). In Section 3, the $n$-species competition model in a general periodic chemostat is studied. Based on Theorem 2.1 , the comparison method and uniformly persistent periodic semiflow theory, sufficient conditions are obtained that guarantee the uniform persistence of all $n$ species and the existence of at least one positive periodic solution of the full system (Theorem 3.1). The special case when the species-specific removal rates of all of the species equal the nutrient dilution rate is also discussed (Corollary 3.2) and an improved result is given when there are only two competing species (Corollary 3.3). Finally, in Section 4, the 3-species competition model is studied under the additional assumptions that the species-specific removal rates of all of the species equal the nutrient dilution rate and that the positive, periodic solutions to each of the three, 2-species subsystems of the limiting 3-species competition system is unique. We determine sufficient conditions, that are more easily satisfied than those given in Section 3, that guarantee the uniform persistence of the three interacting species and prove existence of at least one positive periodic solution for the full model system (Theorems 4.1, 4.2 and 4.3). Theorems 4.2 and 4.3 give sufficient conditions for competition-mediated coexistence. Theorem 4.2 is illustrated by an example.

2. Single population growth. We first consider the nonautonomous Kolmogorov equation on single-species population growth

$$
\frac{d u}{d t}=u F(t, u), \quad u \in R_{+}=[0, \infty),
$$

where $F(t, u): R_{+}^{2} \rightarrow R$ is continuous and locally Lipschitz in $u$. Let $F_{0}(t, u): R_{+}^{2} \rightarrow R$ be continuous, $\omega$-periodic in $t(\omega>0)$ and locally Lipschitz in $u$ uniformly for $t \in[0, \omega]$. Let $\phi(t, s, u), t \geq s \geq 0$, be the unique solution of (2.1) with $\phi(s, s, u)=u$. We assume that

(C1) $\lim _{t \rightarrow \infty}\left|F(t, u)-F_{0}(t, u)\right|=0$ uniformly for $u$ in any bounded subset of $R_{+}$, and that there exists $K>0$ such that $F(t, u) \leq 0$ for all $t \geq 0$ and $u \geq K$ 
(C2) For any $t \in R_{+}, F_{0}(t, u)$ is strictly decreasing for $u$, and that there exists $K_{0}>0$ such that $F_{0}\left(t, K_{0}\right) \leq 0$ for all $t \geq 0$.

Then, we have the following threshold-type result on the global asymptotics for the asymptotically periodic equation (2.1).

Theorem 2.1. Assume that $(\mathrm{C} 1)$ and $(\mathrm{C} 2)$ hold.

(a) If $\int_{0}^{\omega} F_{0}(t, 0) d t \leq 0$, then for any $u \in R_{+}, \lim _{t \rightarrow \infty} \phi(t, 0, u)=0$;

(b) If $\int_{0}^{\omega} F_{0}(t, 0) d t>0$, then for any $u \in R_{+} \backslash\{0\}, \lim _{t \rightarrow \infty}(\phi(t, 0, u)-$ $\left.u^{*}(t)\right)=0$, where $u^{*}(t)$ is the unique positive $\omega$-periodic solution of the periodic Kolmogorov equation $\frac{d u}{d t}=u F_{0}(t, u)$.

Proof. Let $\phi_{0}(t, s, u), t \geq s \geq 0$, be the unique solution of the $\omega$-periodic Kolmogorov equation

$$
\frac{d u}{d t}=u F_{0}(t, u), \quad u \in R_{+},
$$

with $\phi_{0}(s, s, u)=u \in R_{+}$. We first claim that the following threshold result on the global asymptotics of (2.2) holds.

(i) If $\int_{0}^{\omega} F_{0}(t, 0) d t \leq 0$, then for any $u \in R_{+}, \lim _{t \rightarrow \infty} \phi_{0}(t, 0, u)=0$.

(ii) If $\int_{0}^{\omega} F_{0}(t, 0) d t>0$, then (2.2) admits a unique positive periodic solution $u^{*}(t)$ and for any $u \in R_{+} \backslash\{0\}, \lim _{t \rightarrow \infty}\left(\phi_{0}(t, 0, u)-u^{*}(t)\right)=0$.

Indeed, by Zhao and Hutson [22, Lemmas 3.2 and 3.3], it remains to prove the conclusion in the critical case $\int_{0}^{\omega} F_{0}(t, 0) d t=0$. For any $u>0, u(t)=$ $\phi_{0}(t, 0, u)>0$ for all $t>0$, and by the strict monotonicity of $F(t, u)$ for $u \geq 0$,

$$
\frac{d u}{d t}=u(t) F(t, u(t))<u(t) F(t, 0), \quad \text { for all } \quad t>0 .
$$

Then, by the comparison theorem, $u(t)<u(0) e^{\int_{0}^{t} F(s, 0) d s}$, for all $t>0$, and hence the Poincaré map $Q: R_{+} \rightarrow R_{+}$defined by $Q(u)=u(\omega)$ satisfies

$$
Q(u)=u(\omega)<u(0) e^{\int_{0}^{\omega} F(s, 0) d s}=u(0)=u,
$$

which implies that $Q: R_{+} \rightarrow R_{+}$admits no positive fixed point, and that for any $u>0,0<Q^{n+1}(u)<Q^{n}(u), n \geq 0$. Therefore, there exists $\bar{u} \geq 0$ such that $\lim _{n \rightarrow \infty} Q^{n}(u)=\bar{u}$. Since $\bar{u}=Q(\bar{u})$, the nonexistence of positive fixed points of $Q$ implies $\bar{u}=0$. Then, for any $u>0, \lim _{n \rightarrow \infty} Q^{n}(u)=0$, and hence $\lim _{t \rightarrow \infty} u(t)=0$.

Under the continuous differentiability assumption on $F(t, u)$ with respect to $u$, as a simple corollary of Zhao [19, Theorem 3.5], the threshold result on the global asymptotic stability holds for (2.2). 
From conditions (C1) and (C2), it easily follows that for any $u \in R_{+}$and any $s \geq 0, \phi(t, s, u)$ and $\phi_{0}(t, s, u)$ exist globally on $[s, \infty)$, and solutions of (2.1) and (2.2) are uniformly bounded. By [21, Proposition 3.2], $\phi(t, s, u)$ is asymptotic to the $\omega$-periodic semiflow $T(t)=\phi_{0}(t, 0, \cdot): R_{+} \rightarrow R_{+}$, and hence $T_{n}(u)=\phi(n \omega, 0, u), n \geq 0$, is an asymptotically autonomous discrete dynamical process with limiting discrete semiflow $Q^{n}: R_{+} \rightarrow$ $R_{+}, n \geq 0$, where $Q=T(\omega)$ is the Poincaré map associated with the periodic equation (2.2). By [21, Theorem 3.1], it suffices to prove in case (a) that $\lim _{n \rightarrow \infty} T_{n}(u)=0$ for any $u \in R_{+}$, and in case (b) that $\lim _{n \rightarrow \infty} T_{n}(u)=$ $u^{*}(0)$ for any $u \in R_{+} \backslash\{0\}$.

In case (a), by conclusion (i) above, $u=0$ is a globally attractive fixed point of $Q$ in $R_{+}$, and hence $u=0$ is a unique isolated fixed point of $Q$ and is $Q$-acyclic in $R_{+}$. Therefore, for any $u \in R_{+}$, by the convergence theorem in [21, Theorem 2.4], $\omega(u)=0$; i.e., $\lim _{n \rightarrow \infty} T_{n}(u)=0$.

In case (b), by conclusion (ii) above, $u=u^{*}(0)$ is a globally attractive fixed point of $Q$ in $R_{+} \backslash\{0\}$, and hence the only fixed points of $Q$ in $R_{+}$ are 0 and $u^{*}(0)$; both are isolated and there is no $Q$-cyclic chain among them. Therefore, for any $u \in R_{+}$, again by the convergence theorem in [21, Theorem 2.4], either $\omega(u)=0$ or $\omega(u)=u^{*}(0)$. By Lemma 2 in the Appendix, with $m=1, \widetilde{W^{s}}(0) \cap\left(R^{+} \backslash\{0\}\right)=\emptyset$; i.e., for any $u>0, \omega(u) \neq 0$. Therefore, for any $u>0, \omega(u)=u^{*}(0)$; i.e., $\lim _{n \rightarrow \infty} T_{n}(u)=u^{*}(0)$. This completes the proof.

Now consider a single population growth model in a periodic chemostat

$$
\begin{aligned}
& \frac{d S(t)}{d t}=\left(S^{0}(t)-S(t)\right) D_{0}(t)-x(t) P(t, S(t)), \\
& \frac{d x(t)}{d t}=x(t)\left(P(t, S(t))-D_{1}(t)\right) .
\end{aligned}
$$

Here $S(t)$ denotes the concentration of the nutrient, $x(t)$ denotes the biomass of species at time $t, P(t, s)$ represents the specific per capita nutrient uptake function, $S^{0}(t)$ and $D_{0}(t)$ are the input nutrient concentration and the dilution rate respectively, and $D_{1}(t)$ represents the specific removal rate of the species. We assume that $S^{0}(t), D_{0}(t)$ and $D_{1}(t)$ are all continuous, $\omega$-periodic, positive functions, and that $P(t, s): R_{+}^{2} \rightarrow R_{+}$is continuous, $\omega$-periodic in $t$ and satisfies

(i) $P(t, s)$ is locally Lipschitz in $s$; and

(ii) $P(t, 0)=0, t \geq 0$, and for any $t \geq 0, P(t, s)$ is strictly increasing for $s \in R_{+}$.

Let $D(t): R_{+} \rightarrow R_{+}$be a continuous, $\omega$-periodic and positive function. 
For the linear periodic equation

$$
\frac{d V(t)}{d t}=S^{0}(t) D_{0}(t)-D(t) V(t)
$$

it easily follows that (2.4) admits a unique positive $\omega$-periodic solution $V^{*}(t)$ such that every solution $V(t)$ of $(2.4)$ with $V(0) \geq 0$ satisfies $\lim _{t \rightarrow \infty}(V(t)-$ $\left.V^{*}(t)\right)=0$. Moreover, $V^{*}(t)$ can be expressed explicitly as $V^{*}(t)=$

$$
e^{-\int_{0}^{t} D(s) d s}\left[\frac{\int_{0}^{\omega} e^{\int_{0}^{s} D(u) d u} S^{0}(s) D_{0}(s) d s}{e^{\int_{0}^{\omega} D(s) d s}-1}+\int_{0}^{t} e^{\int_{0}^{s} D(u) d u} S^{0}(s) D_{0}(s) d s\right] .
$$

Let $\bar{D}(t)=\max \left(D_{0}(t), D_{1}(t)\right)$ and $\underline{D}(t)=\min \left(D_{0}(t), D_{1}(t)\right)$. Then, $\bar{D}(t)$ and $\underline{D}(t): R_{+} \rightarrow R_{+}$are continuous, $\omega$-periodic and positive functions. Let $V_{1}^{*}(t)$ and $V_{2}^{*}(t)$ be the unique positive $\omega$-periodic solutions of (2.4) with $D(t)$ replaced by $\underline{D}(t)$ and $\bar{D}(t)$ respectively. By the comparison theorem and the global attractivity of $V_{i}^{*}(t)(i=1,2)$, it easily follows that $V_{2}^{*}(t) \leq V_{1}^{*}(t)$ for all $t \geq 0$.

Theorem 2.2. (a) If $\int_{0}^{\omega}\left(P\left(t, V_{2}^{*}(t)\right)-D_{1}(t)\right) d t>0$, then system (2.3) admits a positive (componentwise) $\omega$-periodic solution, and there exist $\alpha>0$ and $\beta>0$ such that every solution $(S(t), x(t))$ of $(2.3)$ with $S(0) \geq 0$ and $x(0)>0$ satisfies

$$
\alpha \leq \liminf _{t \rightarrow \infty} x(t) \leq \limsup _{t \rightarrow \infty} x(t) \leq \beta .
$$

(b) If $\int_{0}^{\omega}\left(P\left(t, V_{1}^{*}(t)\right)-D_{1}(t)\right) d t \leq 0$, then every solution $(S(t), x(t))$ of $(2.3)$ with $S(0) \geq 0$ and $x(0) \geq 0$ satisfies $\lim _{t \rightarrow \infty} x(t)=0$.

Interpreting the predictions of the model biologically, Theorem 2.2 implies that in case (a) the model system admits a periodic coexistence state and the species is uniformly persistent, but in case (b) the species ultimately goes to extinction.

Proof. Let $\hat{P}(t, s): R_{+} \times R \rightarrow R$ be any given continuous extension of $P(t, s)$ on $R_{+} \times R_{+}$to $R_{+} \times R$ such that $\hat{P}(t, s)$ is $\omega$-periodic in $t$ and locally Lipschitz in $s$, and for any $t \geq 0, \hat{P}(t, s)$ is strictly increasing for $s \in R$.

In case (a), since $V_{1}^{*}(t) \geq V_{2}^{*}(t), t \in[0, \omega]$, and $\hat{P}\left(t, V_{i}^{*}(t)\right)=P\left(t, V_{i}^{*}(t)\right)$, $t \in[0, \omega](i=1,2)$, by Theorem 2.1 (in the periodic case), the periodic equation

$$
\frac{d x(t)}{d t}=x(t)\left(\hat{P}\left(t, V_{i}^{*}(t)-x(t)\right)-D_{1}(t)\right)
$$

admits a unique positive $\omega$-periodic solution $x_{i}^{*}(t)$, and $x_{i}^{*}(t)$ is globally attractive in $R_{+} \backslash\{0\}(\mathrm{i}=1,2)$. By the comparison theorem, it easily follows 
that $x_{1}^{*}(t) \geq x_{2}^{*}(t), t \in[0, \omega]$. We further claim that $V_{1}^{*}(t)>x_{1}^{*}(t), t \in[0, \omega]$. Indeed, let $x_{1}^{*}\left(t_{1}\right)=\max _{0 \leq t \leq \omega} x_{1}^{*}(t), t_{1} \in[0, \omega]$; then $\frac{d x_{1}^{*}\left(t_{1}\right)}{d t}=0$, and hence

$$
\hat{P}\left(t_{1}, V_{1}^{*}\left(t_{1}\right)-x_{1}^{*}\left(t_{1}\right)\right)=D_{1}\left(t_{1}\right)>0 .
$$

Since $\hat{P}\left(t_{1}, s\right)$ is strictly increasing for $s \in R, V_{1}^{*}\left(t_{1}\right)>x_{1}^{*}\left(t_{1}\right)$. Let $y(t)=$ $V_{1}^{*}(t)-x_{1}^{*}(t)$. Then, $y(t)$ satisfies the following periodic differential equation:

$$
\frac{d y}{d t}=S^{0}(t) D_{0}(t)-\underline{D}(t) V_{1}^{*}(t)-\left(V_{1}^{*}(t)-y\right)\left(\hat{P}(t, y)-D_{1}(t)\right) .
$$

Since $y\left(t_{1}\right)>0$ and

$$
\left.\frac{d y}{d t}\right|_{y=0}=S^{0}(t) D_{0}(t)+\left(D_{1}(t)-\underline{D}(t)\right) V_{1}^{*}(t) \geq S^{0}(t) D_{0}(t)>0,
$$

$y(t)>0$ for all $t \geq t_{1}$. Then, the $\omega$-periodicity of $y(t)$ implies that $y(t)>0$ for all $t \geq 0$; i.e., $V_{1}^{*}(t)>x_{1}^{*}(t)$ for all $t \geq 0$.

For any $\left(S_{0}, x_{0}\right) \in R_{+}^{2}$ with $S_{0} \geq 0$ and $x_{0}>0$, let $(S(t), x(t))$ be the unique solution of (2.3) satisfying $S(0)=S_{0}$ and $x(0)=x_{0}$ with $[0, \beta)$ as its maximal existence interval. Then, it easily follows that $S(t)>0$ and $x(t)>0$ for all $t \in(0, \beta)$. Let $V(t)=S(t)+x(t)$; then,

$$
S^{0}(t) D_{0}(t)-\bar{D}(t) V(t) \leq \frac{d V(t)}{d t} \leq S^{0}(t) D_{0}(t)-\underline{D}(t) V(t), \quad t \in[0, \beta) .
$$

Let $\bar{V}(t)$ be the unique solution of the linear $\omega$-periodic equation

$$
\frac{d V}{d t}=S^{0}(t) D_{0}(t)-\underline{D}(t) V
$$

satisfying $\bar{V}(0)=V(0)$, and let $\underline{V}(t)$ be the unique solution of the linear $\omega$-periodic equation

$$
\frac{d V}{d t}=S^{0}(t) D_{0}(t)-\bar{D}(t) V
$$

satisfying $\underline{V}(0)=V(0)$. Then, by the standard comparison theorem,

$$
\underline{V}(t) \leq V(t) \leq \bar{V}(t), \quad t \in[0, \beta) .
$$

Since $\bar{V}(t)$ and $\underline{V}(t)$ exist globally on $[0, \infty), \beta=\infty$. Therefore, $x(t)$ satisfies

$$
x(t)\left(\hat{P}(t, \underline{V}(t)-x(t))-D_{1}(t)\right) \leq \frac{d x(t)}{d t} \leq x(t)\left(\hat{P}(t, \bar{V}(t)-x(t))-D_{1}(t)\right),
$$


for all $t \geq 0$. Then, by the comparison theorem,

$$
\underline{x}(t) \leq x(t) \leq \bar{x}(t), \quad t \geq 0,
$$

where $\bar{x}(t)$ is the unique solution of the nonautonomous equation

$$
\frac{d x(t)}{d t}=x(t)\left(\hat{P}(t, \bar{V}(t)-x(t))-D_{1}(t)\right)
$$

with $\bar{x}(0)=x_{0}$, and $\underline{x}(t)$ is the unique solution of the nonautonomous equation

$$
\frac{d x(t)}{d t}=x(t)\left(\hat{P}(t, \underline{V}(t)-x(t))-D_{1}(t)\right),
$$

with $\underline{x}(0)=x_{0}$. Since $\lim _{t \rightarrow \infty}\left(\bar{V}(t)-V_{1}^{*}(t)\right)=0$ and $\lim _{t \rightarrow \infty}\left(\underline{V}(t)-V_{2}^{*}(t)\right)=$ 0 ,

$$
\lim _{t \rightarrow \infty}\left(\hat{P}(t, \bar{V}(t)-x)-\hat{P}\left(t, V_{1}^{*}(t)-x\right)\right)=0
$$

and

$$
\lim _{t \rightarrow \infty}\left(\hat{P}(t, \underline{V}(t)-x)-\hat{P}\left(t, V_{2}^{*}(t)-x\right)\right)=0,
$$

uniformly for $x$ in any bounded subset of $R_{+}$. In case (a), since

$$
\begin{aligned}
\int_{0}^{\omega}\left(\hat{P}\left(t, V_{1}^{*}(t)\right)-D_{1}(t)\right) d t & \geq \int_{0}^{\omega}\left(\hat{P}\left(t, V_{2}^{*}(t)\right)-D_{1}(t)\right) d t \\
& =\int_{0}^{\omega}\left(P\left(t, V_{2}^{*}(t)\right)-D_{1}(t)\right) d t>0
\end{aligned}
$$

by Theorem 2.1 (b),

$$
\lim _{t \rightarrow \infty}\left(\bar{x}(t)-x_{1}^{*}(t)\right)=0 \quad \text { and } \quad \lim _{t \rightarrow \infty}\left(\underline{x}(t)-x_{2}^{*}(t)\right)=0 .
$$

Therefore, by (2.7), it follows that

$$
\begin{aligned}
& \liminf _{t \rightarrow \infty}\left(x(t)-x_{2}^{*}(t)\right) \geq \lim _{t \rightarrow \infty}\left(\underline{x}(t)-x_{2}^{*}(t)\right)=0 \\
& \limsup _{t \rightarrow \infty}\left(x(t)-x_{1}^{*}(t)\right) \leq \lim _{t \rightarrow \infty}\left(\bar{x}(t)-x_{1}^{*}(t)\right)=0,
\end{aligned}
$$

and hence there exist $\alpha>0$ and $\beta>0$ such that $x(t)$ satisfies

$$
\alpha \leq \liminf _{t \rightarrow \infty} x(t) \leq \limsup _{t \rightarrow \infty} x(t) \leq \beta
$$


In case $(b)$, since

$$
\begin{aligned}
\int_{0}^{\omega}\left(\hat{P}\left(t, V_{2}^{*}(t)\right)-D_{1}(t)\right) d t & \leq \int_{0}^{\omega}\left(\hat{P}\left(t, V_{1}^{*}(t)\right)-D_{1}(t)\right) d t \\
& =\int_{0}^{\omega}\left(P\left(t, V_{1}^{*}(t)\right)-D_{1}(t)\right) d t \leq 0,
\end{aligned}
$$

by Theorem $2.1(\mathrm{a}), \lim _{t \rightarrow \infty} \bar{x}(t)=0$ and $\lim _{t \rightarrow \infty} \underline{x}(t)=0$, and hence, by (2.7), $\lim _{t \rightarrow \infty} x(t)=0$.

In case (a), it remains to prove the existence of a positive periodic solution of (2.3). Under the abstract setting of periodic semiflows, this can be done by using [20, Theorem 2.3] as in the latter part of the proof of Theorem 3.1. Instead, we give an alternative, more elementary proof. Let $V=S+x$; then, the system (2.3) is transformed into the following $\omega$-periodic system:

$$
\begin{aligned}
\frac{d V}{d t} & =S^{0}(t) D_{0}(t)-D_{0}(t)(V-x)-D_{1}(t) x, \\
\frac{d x}{d t} & =x\left(\hat{P}(t, V-x)-D_{1}(t)\right) .
\end{aligned}
$$

Then, the positive invariance of $R_{+}^{2}$ with respect to (2.3) implies that the closed convex set $W=\{(V, x): V \geq x \geq 0\} \subset R_{+}^{2}$ is positively invariant with respect to (2.12). Moreover, for any $S_{0} \geq 0$ and $x_{0}>0$, since the first equation of (2.3) implies that $\left.\frac{d S(t)}{d t}\right|_{S=0}=S^{0}(t) D_{0}(t)>0$, the unique solution $(S(t), x(t))$ of $(2.3)$ with $S(0)=S_{0}$ and $x(0)=x_{0}$ satisfies $S(t)>0$ and $x(t)>0$ for all $t>0$. That is, for any $V_{0} \geq x_{0}>0$, the unique solution $(V(t), x(t))$ of $(2.12)$ with $V(0)=V_{0}$ and $x(0)=x_{0}$ satisfies $V(t)>x(t)>0$, for all $t>0$. Let $G: W \rightarrow W$ be the Poincaré map associated with (2.12); i.e., for every $\left(V_{0}, x_{0}\right) \in W, G\left(V_{0}, x_{0}\right)=(V(\omega), x(\omega))$. Clearly, the continuous dependence of solutions on initial data implies that $G: W \rightarrow W$ is continuous. Let

$$
W_{0}=\left\{(V, x) \in W: V_{2}^{*}(0) \leq V \leq V_{1}^{*}(0), x_{2}^{*}(0) \leq x \leq x_{1}^{*}(0)\right\} .
$$

Since $0<x_{1}^{*}(t)<V_{1}^{*}(t), t \in[0, \omega],\left(V_{1}^{*}(0), x_{1}^{*}(0)\right)$ is in the interior of $W$, and hence $W_{0}$ is a nonempty, closed, bounded and convex subset of $R_{+}^{2}$. For any $\left(V_{0}, x_{0}\right) \in W_{0}$, the corresponding solution $(V(t), x(t))$ of $(2.12)$ with $V(0)=V_{0}$ and $x(0)=x_{0}$ satisfies

$$
(V(t), x(t)) \in W \quad \text { for all } \quad t \geq 0
$$

that is, $V(t) \geq x(t) \geq 0$ for all $t \geq 0$. Then, $V(t)$ satisfies

$$
S^{0}(t) D_{0}(t)-\bar{D}(t) V(t) \leq \frac{d V(t)}{d t} \leq S^{0}(t) D_{0}(t)-\underline{D}(t) V(t), \quad t \geq 0 .
$$


Since $V_{2}^{*}(0) \leq V_{0} \leq V_{1}^{*}(0)$, by the comparison theorem,

$$
V_{2}^{*}(t) \leq V(t) \leq V_{1}^{*}(t), \quad t \geq 0 .
$$

Therefore, $x(t)$ satisfies

$$
x(t)\left(\hat{P}\left(t, V_{2}^{*}(t)-x(t)\right)-D_{1}(t)\right) \leq \frac{d x(t)}{d t}, \quad t \geq 0,
$$

and

$$
\frac{d x(t)}{d t} \leq x(t)\left(\hat{P}\left(t, V_{1}^{*}(t)-x(t)\right)-D_{1}(t)\right), \quad t \geq 0 .
$$

Since $x_{2}^{*}(0) \leq x_{0} \leq x_{1}^{*}(0)$, again by the comparison theorem,

$$
x_{2}^{*}(t) \leq x(t) \leq x_{1}^{*}(t), \quad t \geq 0 .
$$

Therefore, (2.14) and (2.15) imply that

$$
\begin{gathered}
V_{2}^{*}(0)=V_{2}^{*}(\omega) \leq V(\omega) \leq V_{1}^{*}(\omega)=V_{1}^{*}(0), \\
x_{2}^{*}(0)=x_{2}^{*}(\omega) \leq x(\omega) \leq x_{1}^{*}(\omega)=x_{1}^{*}(0) .
\end{gathered}
$$

Then, by (2.13) and (2.16), $G\left(V_{0}, x_{0}\right)=(V(\omega), x(\omega)) \in W_{0}$, and hence $G\left(W_{0}\right) \subset W_{0}$. By the Brouwer fixed-point theorem, it follows that there exists $\left(V^{*}, x^{*}\right) \in W_{0}$, such that $G\left(V^{*}, x^{*}\right)=\left(V^{*}, x^{*}\right)$. Clearly, the unique solution $\left(V^{*}(t), x^{*}(t)\right)$ of $(2.12)$ with $\left(V^{*}(0), x^{*}(0)\right)=\left(V^{*}, x^{*}\right)$ is an $\omega$ periodic solution of (2.12). Since $V^{*} \geq x^{*}>0$, by our previous claim, $V^{*}(t)>x^{*}(t)>0$ for all $t>0$, and hence, by the $\omega$-periodicity of $V^{*}(t)$ and $x^{*}(t), V^{*}(t)>x^{*}(t)>0$ for all $t \geq 0$. Therefore, $\left(S^{*}(t), x^{*}(t)\right)=$ $\left(V^{*}(t)-x^{*}(t), x^{*}(t)\right)$ is a positive (componentwise), $\omega$-periodic solution of system (2.3). This completes the proof.

In the case that $D_{0}(t)=D_{1}(t), t \in[0, \omega], V_{1}^{*}(t)=V_{2}^{*}(t), x_{1}^{*}(t)=x_{2}^{*}(t)$, $t \in[0, \omega]$, and hence, by (2.10) and (2.11) in the proof of Theorem 2.2, we have the following threshold-type result on the global dynamics of the model system.

Corollary 2.3. Let $D_{0}(t)=D_{1}(t), t \in[0, \omega]$.

(a) If $\int_{0}^{\omega}\left(P\left(t, V_{1}^{*}(t)\right)-D_{1}(t)\right)>0$, then system (2.3) admits a unique positive, periodic solution $\left(S^{*}(t), x_{1}^{*}(t)\right)=\left(V_{1}^{*}(t)-x_{1}^{*}(t), x_{1}^{*}(t)\right)$, and any solution $(S(t), x(t))$ of $(2.3)$ with $S(0) \geq 0$ and $x(0)>0$ satisfies $\lim _{t \rightarrow \infty}\left(S(t)-S^{*}(t)\right)=0$ and $\lim _{t \rightarrow \infty}\left(x(t)-x^{*}(t)\right)=0$.

(b) If $\int_{0}^{\omega}\left(P\left(t, V_{1}^{*}(t)\right)-D_{1}(t)\right) \leq 0$, then any solution $(S(t), x(t))$ of $(2.3)$ with $S(0) \geq 0$ and $x(0) \geq 0$ satisfies $\lim _{t \rightarrow \infty}\left(S(t)-V_{1}^{*}(t)\right)=0$ and $\lim _{t \rightarrow \infty} x(t)=0$. 
3. n-Species competition. In this section, we consider the n-species competition model in the periodic chemostat

$$
\begin{aligned}
& \frac{d S(t)}{d t}=\left(S^{0}(t)-S(t)\right) D_{0}(t)-\sum_{i=1}^{n} P_{i}(t, S(t)) x_{i}(t), \\
& \frac{d x_{i}(t)}{d t}=x_{i}(t)\left(P_{i}(t, S(t))-D_{i}(t)\right), \quad 1 \leq i \leq n .
\end{aligned}
$$

Here $S(t)$ denotes the concentration of the nutrient, $x_{i}(t)$ denotes the biomass of the i-th species at time $t, P_{i}(t, s)$ represents the specific per capita nutrient uptake function of the i-th species, $S^{0}(t)$ and $D_{0}(t)$ are the input nutrient concentration and the dilution rate respectively, and $D_{i}(t)$ represents the specific removal rate or washout rate of species $x_{i}$. We assume that $S^{0}(t)$ and $D_{i}(t)(1 \leq i \leq n)$ are all continuous, $\omega$-periodic and positive functions, and that each $P_{i}(t, s)(1 \leq i \leq n)$ satisfies the same conditions as $P(t, s)$ in Section 2. Let

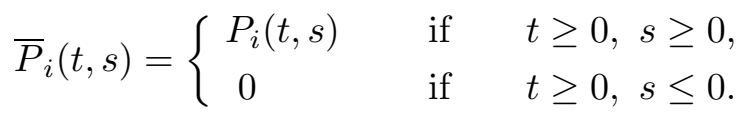

Then, $\bar{P}_{i}: R_{+} \times R \rightarrow R$ is a continuous extension of $P_{i}(t, s)$ on $R_{+} \times R_{+}$ to $R_{+} \times R(1 \leq i \leq n)$. Let $\bar{D}(t)=\max \left(D_{0}(t), D_{1}(t), \ldots, D_{n}(t)\right)$ and $\underline{D}(t)=\min \left(D_{0}(t), D_{1}(t), \ldots, D_{n}(t)\right)$. Then, $\bar{D}(t)$ and $\underline{D}(t): R_{+} \rightarrow R_{+}$are continuous, $\omega$-periodic, and positive functions. Let $V_{1}^{*}(t)$ and $V_{2}^{*}(t)$ be the unique positive $\omega$-periodic solutions of $(2.4)$ with $D(t)$ replaced by $\underline{D}(t)$ and $\bar{D}(t)$ respectively. As shown in Section $2, V_{2}^{*}(t) \leq V_{1}^{*}(t)$ for all $t \geq 0$.

We are now in a position to prove the main result of this section.

Theorem 3.1. Assume that

(1) $\int_{0}^{\omega}\left(P_{i}\left(t, V_{1}^{*}(t)\right)-D_{i}(t)\right) d t>0, \quad 1 \leq i \leq n$;

(2) $\int_{0}^{\omega}\left(\bar{P}_{i}\left(t, V_{2}^{*}(t)-\sum_{j=1, j \neq i}^{n} x_{j}^{*}(t)\right)-D_{i}(t)\right) d t>0,1 \leq i \leq n$, where $x_{j}^{*}(t)$ is the unique positive $\omega$-periodic solution of the scalar periodic equation $\frac{d x_{j}}{d t}=x_{j}\left(P_{j}\left(t, V_{1}^{*}(t)-x_{j}\right)-D_{j}(t)\right), 1 \leq j \leq n$.

Then, system (3.1) admits a positive $\omega$-periodic solution, and there exist $\alpha>0$ and $\beta>0$ such that any solution $\left(S(t), x_{1}(t), \ldots, x_{n}(t)\right)$ of $(3.1)$ with $S(0) \geq 0$ and $x_{i}(0)>0(1 \leq i \leq n)$ satisfies

$$
0<\alpha \leq \liminf _{t \rightarrow \infty} x_{i}(t) \leq \limsup _{t \rightarrow \infty} x_{i}(t) \leq \beta, \quad 1 \leq i \leq n
$$

Proof. Let $\hat{P}_{i}(t, s): R_{+} \times R \rightarrow R$ be any given continuous extension of $P_{i}(t, s)$ on $R_{+} \times R_{+}$to $R_{+} \times R$, such that $\hat{P}_{i}(t, s)$ is $\omega$-periodic in $t$ and 
locally Lipschitz in $s$, and for any $t \geq 0, \hat{P}_{i}(t, s)$ is strictly increasing with respect to $s \in R, 1 \leq i \leq n$. By Theorem 2.1 (in the periodic case), condition (1) implies that for each $1 \leq i \leq n$, the periodic equation

$$
\frac{d x_{i}}{d t}=x_{i}\left(\hat{P}_{i}\left(t, V_{1}^{*}(t)-x_{i}\right)-D_{i}(t)\right)
$$

admits a unique $\omega$-periodic solution $x_{i}^{*}(t)$ and $x_{i}^{*}(t)$ is globally attractive in $R_{+} \backslash\{0\}$. As in the proof of Theorem 2.2, $V_{1}^{*}(t)>x_{i}^{*}(t), t \in[0, \omega]$. Then, $x_{i}^{*}(t)$ is a unique positive $\omega$-periodic solution of the periodic equation

$$
\frac{d x_{i}}{d t}=x_{i}\left(P_{i}\left(t, V_{1}^{*}(t)-x_{i}\right)-D_{i}(t)\right)
$$

that is, $x_{i}^{*}(t)$ is independent of the choice of the extension $\hat{P}_{i}(t, s)$ of $P_{i}(t, s)$. Let

$$
\hat{P}_{i \epsilon}(t, s)=\left\{\begin{array}{cc}
P_{i}(t, s) & \text { if } t \geq 0, s \geq 0 \\
\epsilon s & \text { if } t \geq 0, s \leq 0 .
\end{array}\right.
$$

By the boundedness of $V_{2}^{*}(t)-\sum_{j=1, j \neq i}^{n} x_{j}^{*}(t)$ on $[0, \infty)$, it easily follows that

$\lim _{\epsilon \rightarrow 0^{+}} \int_{0}^{\omega} \hat{P}_{i \epsilon}\left(t, V_{2}^{*}(t)-\sum_{j=1, j \neq i}^{n} x_{j}^{*}(t)\right) d t=\int_{0}^{\omega}\left(\bar{P}_{i}\left(t, V_{2}^{*}(t)-\sum_{j=1, j \neq i}^{n} x_{j}^{*}(t)\right)\right) d t$

$(1 \leq i \leq n)$. Then, by condition $(2)$, there exists $\epsilon>0$ such that

$$
\int_{0}^{\omega}\left(\hat{P}_{i \epsilon}\left(t, V_{2}^{*}(t)-\sum_{j=1, j \neq i}^{n} x_{j}^{*}(t)\right)-D_{i}(t)\right) d t>0,1 \leq i \leq n .
$$

In what follows, for simplicity, we denote $\hat{P}_{i \epsilon}(t, s)$ by $\hat{P}_{i}(t, s)(1 \leq i \leq n)$.

For any $\left(S_{0}, x_{0}\right)=\left(S_{0}, x_{1}^{0}, \ldots, x_{n}^{0}\right) \in R_{+}^{n+1}$ with $x_{i}^{0}>0(1 \leq i \leq n)$, let $(S(t), x(t))=\left(S(t), x_{1}(t), \ldots, x_{n}(t)\right)$ be the unique solution of (3.1) satisfying $S(0)=S_{0}, x(0)=x_{0}$ on the maximal existence interval $[0, \beta)$. Since $\left.\frac{d S}{d t}\right|_{S=0}=S^{0}(t) D_{0}(t)>0, S(t)>0$ and $x(t)>0$ for all $t \in[0, \beta)$. Let $V(t)=S(t)+\sum_{i=1}^{n} x_{i}(t)$; then,

$$
S^{0}(t) D_{0}(t)-\bar{D}(t) V(t) \leq \frac{d V(t)}{d t} \leq S^{0}(t) D_{0}(t)-\underline{D}(t) V(t) .
$$

Therefore, by the comparison theorem,

$$
\underline{V}(t) \leq V(t) \leq \bar{V}(t), \quad t \in[0, \beta),
$$


where $\bar{V}(t)$ is the unique solution of the linear $\omega$-periodic equation

$$
\frac{d V}{d t}=S^{0}(t) D_{0}(t)-\underline{D}(t) V(t)
$$

with $\bar{V}(0)=V(0)$, and $\underline{V}(t)$ is the unique solution of the linear $\omega$-periodic equation

$$
\frac{d V}{d t}=S^{0}(t) D_{0}(t)-\bar{D}(t) V(t)
$$

with $\underline{V}(0)=V(0)$, respectively. The global existence of $\bar{V}(t)$ on $[0, \infty)$ implies that $\beta=\infty$. Since $\lim _{t \rightarrow \infty}\left(\bar{V}(t)-V_{1}^{*}(t)\right)=0, V(t)$, and hence $S(t)$ and $x(t)=\left(x_{1}(t), \ldots, x_{n}(t)\right)$ are ultimately bounded. That is, system (3.1) is point dissipative on $R_{+}^{n+1}$. Therefore, for all $t \geq 0,1 \leq i \leq n$,

$$
\begin{aligned}
\frac{x_{i}(t)}{d t} & =x_{i}(t)\left(\hat{P}_{i}\left(t, V(t)-\sum_{j=1}^{n} x_{j}(t)\right)-D_{i}(t)\right) \\
& \leq x_{i}(t)\left(\hat{P}_{i}\left(t, V(t)-x_{i}(t)\right)-D_{i}(t)\right) .
\end{aligned}
$$

Then, by the comparison theorem,

$$
x_{i}(t) \leq \bar{x}_{i}(t), \quad 1 \leq i \leq n, \quad t \geq 0,
$$

where $\bar{x}_{i}(t)$ is the unique solution of the nonautonomous equation

$$
\frac{d x_{i}(t)}{d t}=x_{i}(t)\left(\hat{P}_{i}\left(t, \bar{V}(t)-x_{i}\right)-D_{i}(t)\right)
$$

with $\bar{x}_{i}(0)=x_{i}(0)>0(1 \leq i \leq n)$. Since $\lim _{t \rightarrow \infty}\left(\bar{V}(t)-V_{1}^{*}(t)\right)=0$,

$$
\lim _{t \rightarrow \infty}\left(\hat{P}_{i}\left(t, \bar{V}(t)-x_{i}\right)-\hat{P}\left(t, V_{1}^{*}(t)-x_{i}\right)=0\right.
$$

uniformly for $x_{i}$ in any bounded subset of $R_{+}$. Since

$$
\int_{0}^{\omega}\left(\hat{P}_{i}\left(t, V_{1}^{*}(t)\right)-D_{i}(t)\right) d t=\int_{0}^{\omega}\left(P_{i}\left(t, V_{1}^{*}(t)\right)-D_{i}(t)\right) d t>0,
$$

by Theorem 2.1 (b),

$$
\lim _{t \rightarrow \infty}\left(\bar{x}_{i}(t)-x_{i}^{*}(t)\right)=0, \quad 1 \leq i \leq n .
$$


Therefore, by (3.3) and (3.4), for any $1 \leq i \leq n$ and all $t \geq 0$,

$$
\begin{aligned}
\frac{d x_{i}(t)}{d t} & =x_{i}(t)\left(\hat{P}_{i}\left(t, V(t)-\sum_{j=1}^{n} x_{j}(t)\right)-D_{i}(t)\right) \\
& \geq x_{i}(t)\left(\hat{P}_{i}\left(t, \underline{V}(t)-\sum_{j=1, j \neq i}^{n} \bar{x}_{j}(t)-x_{i}(t)\right)-D_{i}(t)\right),
\end{aligned}
$$

and hence, by the comparison theorem,

$$
x_{i}(t) \geq \underline{x}_{i}(t), \quad 1 \leq i \leq n, \quad t \geq 0,
$$

where $\underline{x}_{i}(t)$ is the unique solution of the nonautonomous equation

$$
\frac{d x_{i}}{d t}=x_{i}(t)\left(\hat{P}_{i}\left(t, \underline{V}(t)-\sum_{j=1, j \neq i}^{n} \bar{x}_{j}(t)-x_{i}\right)-D_{i}(t)\right),
$$

with $\underline{x}_{i}(0)=x_{i}(0)(1 \leq i \leq n)$. Since $\lim _{t \rightarrow \infty}\left(\underline{V}(t)-V_{2}^{*}(t)\right)=0$, by $(3.6)$,

$$
\lim _{t \rightarrow \infty}\left(\hat{P}_{i}\left(t, \underline{V}(t)-\sum_{j=1, j \neq i}^{n} x_{j}^{*}(t)-x_{i}\right)-\hat{P}_{i}\left(t, V_{2}^{*}(t)-\sum_{j=1, j \neq i}^{n} x_{j}^{*}(t)-x_{i}\right)\right)=0,
$$

uniformly for $x_{i}$ in any bounded subset of $R_{+}(1 \leq i \leq n)$. Then, by (3.2) and Theorem 2.1 (b),

$$
\lim _{t \rightarrow \infty}\left(\underline{x}_{i}(t)-\underline{x}_{i}^{*}(t)\right)=0,
$$

where $\underline{x}_{i}^{*}(t)(1 \leq i \leq n)$ is the unique positive $\omega$-periodic solution of the periodic equation

$$
\frac{d x_{i}}{d t}=x_{i}(t)\left(\hat{P}_{i}\left(t, V_{2}^{*}(t)-\sum_{j=1, j \neq i}^{n} x_{j}^{*}(t)-x_{i}\right)-D_{i}(t)\right) .
$$

Then, by (3.4), (3.6), (3.8) and (3.10),

$$
\liminf _{t \rightarrow \infty}\left(x_{i}(t)-\underline{x}_{i}^{*}(t) \geq 0 \geq \limsup _{t \rightarrow \infty}\left(x_{i}(t)-x_{i}^{*}(t)\right),\right.
$$

for all $1 \leq i \leq n$. Clearly, (3.12) implies that there exist $\alpha>0$ and $\beta>0$ such that any solution $\left(S(t), x_{1}(t), \ldots, x_{n}(t)\right)$ of $(3.1)$ with $S(0) \geq 0$ and $x_{i}(0)>0(1 \leq i \leq n)$ satisfies

$$
0<\alpha \leq \liminf _{t \rightarrow \infty} x_{i}(t) \leq \limsup _{t \rightarrow \infty} x_{i}(t) \leq \beta, \quad 1 \leq i \leq n .
$$


To prove the existence of a positive $\omega$-periodic solution of (3.1), let $X=$ $R_{+}^{n+1}, X_{0}=\left\{\left(S, x_{1}, \ldots, x_{n}\right) \in R_{+}^{n+1}, x_{i}>0\right.$ for all $\left.1 \leq i \leq n\right\}$ and $\partial X_{0}=\left\{\left(S, x_{1}, \ldots, x_{n}\right) \in R_{+}^{n+1}, x_{i}=0\right.$ for some $\left.1 \leq i \leq n\right\}$. Then, $X=X_{0} \cup \partial X_{0}$. For any $y=\left(S, x_{1}, \ldots, x_{n}\right) \in X$, let $\phi(t, y), t \geq 0$ be the unique solution of $(3.1)$ with $\phi(0, y)=y$. Clearly, $T(t)=\phi(t, \cdot): X \rightarrow X$ is a periodic semiflow $([20])$, and $T(t) X_{0} \subset X_{0}$ for all $t \geq 0$. As we have shown, $T(t)$ is point dissipative (i.e., ultimately bounded) in $X$ and uniformly persistent with respect to $\left(X_{0}, \partial X_{0}\right)$, (i.e., there exists $\eta>0$ such that for any $\left.y \in X_{0}, \liminf _{t \rightarrow \infty} d\left(T(t) y, \partial X_{0}\right) \geq \eta\right)$. Let $Q=T(\omega): X \rightarrow X$ be the Poincaré map associated with (3.1). Then, by Yoshizawa [18, Theorem 8.5], the ultimate boundedness of solutions of a periodic system of ordinary differential equations implies the uniform boundedness of solutions, and hence $Q: X \rightarrow X$ is compact. Therefore, by [20, Theorem 2.3], $Q$ admits a fixed point $y_{0} \in X_{0}$, i.e., $y_{0}=Q\left(y_{0}\right)$, and hence $\phi\left(t, y_{0}\right)$ is a periodic solution of (3.1). Let $y_{0}=\left(S_{0}, x_{1}^{0}, \ldots, x_{n}^{0}\right) \in X_{0}$; then, $S_{0} \geq 0, x_{i}^{0}>0$ for all $1 \leq i \leq n$. It then follows that $\phi\left(t, y_{0}\right)=\left(S(t), x_{1}(t), \ldots, x_{n}(t)\right)$ satisfies $S(t)>0$ and $x_{i}(t)>0(1 \leq i \leq n)$ for all $t>0$. By the $\omega$-periodicity of $\phi\left(t, y_{0}\right), S(t)>0$ and $x_{i}(t)>0(1 \leq i \leq n)$ for all $t \geq 0$. Therefore, $\phi\left(t, y_{0}\right)$ is a positive $\omega$-periodic solution of (3.1). This completes the proof.

In the case $D_{i}(t)=D_{0}(t), t \in[0, \omega], 1 \leq i \leq n, \underline{D}(t)=\bar{D}(t)=D_{0}(t)$, $V_{1}^{*}(t)=V_{2}^{*}(t), t \in[0, \omega]$, and hence we have the following corollary of Theorem 3.1.

Corollary 3.2. Let $D_{i}(t)=D_{0}(t), t \in[0, \omega], 1 \leq i \leq n$. Assume that

(1) $\int_{0}^{\omega}\left(P_{i}\left(t, V_{1}^{*}(t)\right)-D_{0}(t)\right) d t>0, \quad 1 \leq i \leq n$;

(2) $\int_{0}^{\omega}\left(\bar{P}_{i}\left(t, V_{1}^{*}(t)-\sum_{j=1, j \neq i}^{n} x_{j}^{*}(t)\right)-\bar{D}_{0}(t)\right) d t>0,1 \leq i \leq n$, where $x_{j}^{*}(t)$ is the unique positive $\omega$-periodic solution of the scalar periodic equation $\frac{d x_{j}}{d t}=x_{j}\left(P_{j}\left(t, V_{1}^{*}(t)-x_{j}\right)-D_{0}(t)\right), 1 \leq j \leq n$.

Then, system (3.1) admits a positive $\omega$-periodic solution and all $n$ species are uniformly persistent.

As shown in the proof of Theorem 2.2, in the case $D_{i}(t)=D_{0}(t), t \in[0, \omega]$, $1 \leq i \leq n$, it easily follows that $V_{1}^{*}(t)>x_{i}^{*}(t), t \in[0, \omega], 1 \leq i \leq n$. Thus we have the following result for 2 -species competition.

Corollary 3.3. Let $D_{i}(t)=D_{0}(t), t \in[0, \omega], 1 \leq i \leq 2$. Assume that

(1) $\int_{0}^{\omega}\left(P_{i}\left(t, V_{1}^{*}(t)\right)-D_{0}(t)\right) d t>0, \quad 1 \leq i \leq 2$;

(2) $\int_{0}^{\omega}\left(P_{i}\left(t, V_{1}^{*}(t)-x_{j}^{*}(t)\right)-D_{0}(t)\right) d t>0,1 \leq i, j \leq 2, i \neq j$, where $x_{j}^{*}(t)$ is the unique positive $\omega$-periodic solution of the scalar periodic equation $\frac{d x_{j}}{d t}=x_{j}\left(P_{j}\left(t, V_{1}^{*}(t)-x_{j}\right)-D_{0}(t)\right), 1 \leq j \leq 2$. 
Then, system (3.1) admits a positive, $\omega$-periodic solution and both species are uniformly persistent.

Remark 3.4. In [13, Section 7], 2-species competition in the chemostat with periodic dilution rate is discussed in the setting of monotone dynamical systems theory. It is easy to see that our Corollary 3.3 generalizes their result on the uniform persistence of 2 species and the existence of a positive periodic solution for the full system (not just for the limiting system).

4. 3-Species competition. For 2-species competition in a periodic chemostat with $D_{1}(t)=D_{2}(t)=D_{0}(t)(0 \leq t \leq \omega)$, it follows easily that $\left(x_{1}^{*}(t), 0\right)$ and $\left(0, x_{2}^{*}(t)\right)$ are the semitrivial periodic solutions of the limiting 2 -species competition system. Then, condition (2) in Corollary 3.3 is a natural invasibility condition. However, for n-species competition in the periodic chemostat, even with $D_{i}(t)=D_{0}(t)(0 \leq t \leq \omega)(1 \leq i \leq n)$, $\left(x_{1}^{*}(t), \ldots, x_{i-1}^{*}(t), 0, x_{i+1}^{*}(t), \ldots, x_{n}^{*}(t)\right)(1 \leq i \leq n)$ is not the solution of the limiting $\mathrm{n}$-species competition system determined using the conservation principle, and hence, due to our overestimation of the effect of competition, condition (2) in Corollary 3.2 is a stronger invasibility condition than necessary. In this section, we show that whenever the positive, periodic solutions to each of the three, 2 -species subsystems of the limiting 3-species competition system are unique, the expected, natural invasibility conditions are sufficient to guarantee the uniform persistence of the three interacting species and enough to ensure the existence of at least one positive periodic solution for the full model system (see Theorem 4.1). The final results, given in Theorems 4.2 and 4.3, give conditions for competition-mediated coexistence. Here, in at least one of the two-species subsystems, one of the species is driven to extinction, regardless of the initial conditions. However, when the third species is introduced all three species coexist, again independent of the initial conditions provided that they are all positive. We conclude this section with an example that illustrates Theorem 4.2.

Consider the 3 -species competition model in the periodic chemostat

$$
\begin{gathered}
\frac{d S(t)}{d t}=\left(S^{0}(t)-S(t)\right) D_{0}(t)-\sum_{i=1}^{3} P_{i}(t, S(t)) x_{i}(t), \\
\frac{d x_{i}(t)}{d t}=x_{i}(t)\left(P_{i}(t, S(t))-D_{0}(t)\right), \quad 1 \leq i \leq 3 .
\end{gathered}
$$

Here $S^{0}(t), D_{0}(t)$ and $P_{i}(t, s)(1 \leq i \leq 3)$ satisfy the same conditions as in (3.1), with $D_{i}(t)=D_{0}(t)(1 \leq i \leq 3)$. Let $V_{0}^{*}(t)$ be the unique, globally attractive, positive $\omega$-periodic solution of

$$
\frac{d V}{d t}=\left(S^{0}(t)-V(t)\right) D_{0}(t)
$$


For each $1 \leq i \leq 3$, there is a corresponding 2 -species periodic competition system

$$
\frac{d x_{j}}{d t}=x_{j}\left(P_{j}\left(t, V_{0}^{*}-\sum_{k=1, k \neq i}^{3} x_{k}\right)-D_{0}(t)\right), \quad 1 \leq j \leq 3, \quad j \neq i .
$$

We will distinguish among the following three cases:

(A1) Each $\left(E_{i}\right)(1 \leq i \leq 3)$ admits at most one positive $\omega$-periodic solution;

(A2) Each $\left(E_{i}\right)(2 \leq i \leq 3)$ admits at most one positive $\omega$-periodic solution, and $\left(E_{1}\right)$ admits no positive $\omega$-periodic solution;

(A3) $\left(E_{2}\right)$ admits at most one positive $\omega$-periodic solution, and each $\left(E_{i}\right)$ $(i=1,3)$ admits no positive $\omega$-periodic solution.

Theorem 4.1. Let (A1) hold. Assume that

(1) $\mu_{i} \equiv \int_{0}^{\omega}\left(P_{i}\left(t, V_{0}^{*}(t)\right)-D_{0}(t)\right) d t>0, \quad 1 \leq i \leq 3$;

(2) $\mu_{j i} \equiv \int_{0}^{\omega}\left(P_{i}\left(t, V_{0}^{*}(t)-x_{j}^{*}(t)\right)-D_{0}(t)\right) d t>0, \quad 1 \leq i, j \leq 3, i \neq j$;

(3) $\bar{\mu}^{i} \equiv \int_{0}^{\omega}\left(P_{i}\left(t, V_{0}^{*}(t)-\sum_{j=1, j \neq i}^{3} \bar{x}_{j}^{i}(t)\right)-D_{0}(t)\right) d t>0, \quad 1 \leq i \leq 3$, where $x_{i}^{*}(t)(1 \leq i \leq 3)$ is the unique positive, $\omega$-periodic solution of the scalar periodic equation

$$
\frac{d x_{i}}{d t}=x_{i}\left(P_{i}\left(t, V_{0}^{*}(t)-x_{i}\right)-D_{0}(t)\right)
$$

and $\left(\bar{x}_{2}^{1}(t), \bar{x}_{3}^{1}(t)\right),\left(\bar{x}_{1}^{2}(t), \bar{x}_{3}^{2}(t)\right)$ and $\left(\bar{x}_{1}^{3}(t), \bar{x}_{2}^{3}(t)\right)$ are the unique positive, $\omega$-periodic solutions of $\left(E_{1}\right),\left(E_{2}\right)$ and $\left(E_{3}\right)$, respectively.

Then, system (4.1) admits a positive, $\omega$-periodic solution, and there exist $\alpha>0$ and $\beta>0$ such that any solution $\left(S(t), x_{1}(t), x_{2}(t), x_{3}(t)\right)$ of (4.1) with $S(0) \geq 0$ and $x_{i}(0)>0(1 \leq i \leq 3)$ satisfies

$$
0<\alpha \leq \liminf _{t \rightarrow \infty} x_{i}(t) \leq \limsup _{t \rightarrow \infty} x_{i}(t) \leq \beta, \quad 1 \leq i \leq 3
$$

Proof. Let $\hat{P}_{i}(t, s): R_{+} \times R \rightarrow R$ be any given continuous extension of $P_{i}(t, s)$ on $R_{+} \times R_{+}$to $R_{+} \times R$, such that $\hat{P}_{i}(t, s)$ is $\omega$-periodic in $t$ and locally Lipschitz in $s$, and for any $t \geq 0, \hat{P}_{i}(t, s)$ is strictly increasing with respect to $s \in R, 1 \leq i \leq 3$. As in the proof of Theorem 3.1, condition (1) implies that for each $1 \leq i \leq 3$, the periodic equation

$$
\frac{d x_{i}}{d t}=x_{i}\left(P_{i}\left(t, V_{0}^{*}(t)-x_{i}\right)-D_{i}(t)\right)
$$


admits a unique positive, $\omega$-periodic solution $x_{i}^{*}(t)$ with $V_{0}^{*}(t)>x_{i}^{*}(t), t \in$ $[0, \omega]$, and $x_{i}^{*}(t)$ is globally attractive for the periodic equation

$$
\frac{d x_{i}}{d t}=x_{i}\left(\hat{P}_{i}\left(t, V_{0}^{*}(t)-x_{i}\right)-D_{i}(t)\right)
$$

in $R_{+} \backslash\{0\}$. For the 2 -species periodic competition system

$$
\begin{aligned}
& \frac{d x_{1}}{d t}=x_{1}\left(\hat{P}_{1}\left(t, V_{0}^{*}(t)-x_{1}-x_{2}\right)-D_{0}(t)\right), \\
& \frac{d x_{2}}{d t}=x_{2}\left(\hat{P}_{2}\left(t, V_{0}^{*}(t)-x_{1}-x_{2}\right)-D_{0}(t)\right),
\end{aligned}
$$

we claim that if $\left(\tilde{x}_{1}(t), \tilde{x}_{2}(t)\right)$ is a positive $\omega$-periodic solution to $\left(\hat{E}_{3}\right)$, then $\left(\tilde{x}_{1}(t), \tilde{x}_{2}(t)\right)$ satisfies $V_{0}^{*}(t)>\tilde{x}_{1}(t)+\tilde{x}_{2}(t), t \in[0, \omega]$. Indeed, let $\tilde{x}_{1}\left(t_{1}\right)=$ $\max _{0 \leq t \leq \omega} \tilde{x}_{1}(t), t_{1} \in[0, \omega]$; then $\frac{d \tilde{x}_{1}\left(t_{1}\right)}{d t}=0$, and hence $\hat{P}_{1}\left(t_{1}, V_{0}^{*}\left(t_{1}\right)-\tilde{x}_{1}\left(t_{1}\right)-\right.$ $\left.\tilde{x}_{2}\left(t_{1}\right)\right)=D_{0}\left(t_{1}\right)>0$. Then, since $\hat{P}_{1}\left(t_{1}, s\right)$ is strictly increasing for $s \in R$, $V_{0}^{*}\left(t_{1}\right)>\tilde{x}_{1}\left(t_{1}\right)+\tilde{x}_{2}\left(t_{1}\right)$. Let $y(t)=V_{0}^{*}(t)-\tilde{x}_{1}(t)-\tilde{x}_{2}(t)$. It then easily follows that $y(t)$ satisfies the following periodic differential equation:

$$
\frac{d y}{d t}=S^{0}(t) D_{0}(t)-D_{0}(t) y-\tilde{x}_{1}(t) \hat{P}_{1}(t, y)-\tilde{x}_{2}(t) \hat{P}_{2}(t, y) .
$$

Since $y\left(t_{1}\right)>0$ and

$$
\left.\frac{d y}{d t}\right|_{y=0}=S^{0}(t) D_{0}(t)>0,
$$

$y(t)>0$ for all $t \geq t_{1}$. Then, the $\omega$-periodicity of $y(t)$ implies that $y(t)>0$ for all $t \geq 0$; i.e., $V_{0}^{*}(t)>\tilde{x}_{1}(t)+\tilde{x}_{2}(t)$ for all $t \geq 0$. By a standard monotone dynamical systems approach (see, e.g., [13, Chapter 7], [12] and Hess [6, IV.33]), conditions (1) and (2) with $1 \leq i, j \leq 2, i \neq j$ imply that system $\left(\hat{E}_{3}\right)$ is compressive in the sense that there are two positive $\omega$-periodic solutions $\left(\underline{x}_{1}(t), \underline{x}_{2}(t)\right)$ and $\left(\bar{x}_{1}(t), \bar{x}_{2}(t)\right)$ to $\left(\hat{E}_{3}\right)$ with $0<\underline{x}_{1}(t) \leq \bar{x}_{1}(t)$ and $0<\bar{x}_{2}(t) \leq \underline{x}_{2}(t), t \in[0, \omega]$, such that each solution $\left(x_{1}(t), x_{2}(t)\right)$ of $\left(\hat{E}_{3}\right)$ with $x_{1}(0)>0$ and $x_{2}(0)>0$ satisfies

$$
\lim _{t \rightarrow \infty} d\left(x_{1}(t),\left[\underline{x}_{1}(t), \bar{x}_{1}(t)\right]\right)=0 \quad \text { and } \quad \lim _{t \rightarrow \infty} d\left(x_{2}(t),\left[\bar{x}_{2}(t), \underline{x}_{2}(t)\right]\right)=0 .
$$

By our previous claim, $V_{0}^{*}(t)>\underline{x}_{1}(t)+\underline{x}_{2}(t)$ and $V_{0}^{*}(t)>\bar{x}_{1}(t)+\bar{x}_{2}(t)$, and hence both $\left(\underline{x}_{1}(t), \underline{x}_{2}(t)\right)$ and $\left(\bar{x}_{1}(t), \bar{x}_{2}(t)\right)$ are also positive, periodic solutions of $\left(E_{3}\right)$. Therefore, by the uniqueness assumption (A1), $\left(\underline{x}_{1}(t), \underline{x}_{2}(t)\right)=$ $\left(\bar{x}_{1}(t), \bar{x}_{2}(t)\right), t \in[0, \omega]$, and hence $\left(E_{3}\right)$ admits a unique positive, $\omega$-periodic 
solution $\left(\bar{x}_{1}^{3}(t), \bar{x}_{2}^{3}(t)\right)$ with $V_{0}^{*}(t)>\bar{x}_{1}^{3}(t)+\bar{x}_{2}^{3}(t), t \in[0, \omega]$. Furthermore, $\left(\bar{x}_{1}^{3}(t), \bar{x}_{2}^{3}(t)\right)$ is globally attractive for $\left(\hat{E}_{3}\right)$ in $\operatorname{int}\left(R_{+}^{2}\right)$. By a similar argument, it follows that $\left(E_{1}\right)$ and $\left(E_{2}\right)$ admit unique positive, $\omega$-periodic solutions $\left(\bar{x}_{2}^{1}(t), \bar{x}_{3}^{1}(t)\right)$ and $\left(\bar{x}_{1}^{2}(t), \bar{x}_{3}^{2}(t)\right)$ with $V_{0}^{*}(t)>\bar{x}_{2}^{1}(t)+\bar{x}_{3}^{1}(t)$ and $V_{0}^{*}(t)>\bar{x}_{1}^{2}(t)+\bar{x}_{3}^{2}(t), t \in[0, \omega]$, respectively.

For the 3 -species periodic competition system

$$
\frac{d x_{i}}{d t}=x_{i}\left(\hat{P}_{i}\left(t, V_{0}^{*}(t)-\sum_{j=1}^{3} x_{j}\right)-D_{0}(t)\right), \quad 1 \leq i \leq 3,
$$

let $\phi_{0}(t, x)$ be the unique solution of (4.4) with $\phi_{0}(0, x)=x \in R_{+}^{3}$. By a standard comparison theorem argument, it then easily follows that $\phi_{0}(t, x)$ exists globally on $[0, \infty)$ and solutions of $(4.4)$ are uniformly and ultimately bounded. Let $X=R_{+}^{3}$, and let $Q=\phi_{0}(\omega, \cdot)$ be the Poincaré map associated with (4.4). Then, $Q: X \rightarrow X$ is compact and point dissipative. Let $X_{0}=\left\{\left(x_{1}, x_{2}, x_{3}\right) \in R_{+}^{3}, x_{i}>0\right.$ for all $\left.1 \leq i \leq 3\right\}$ and $\partial X_{0}=$ $\left\{\left(x_{1}, x_{2}, x_{3}\right) \in R_{+}^{3}, x_{i}=0\right.$ for some $\left.1 \leq i \leq 3\right\}$. Then, $X=X_{0} \cup \partial X_{0}$. Let $M_{1}=(0,0,0), M_{2}=\left(x_{1}^{*}(0), 0,0\right), M_{3}=\left(0, x_{2}^{*}(0), 0\right), M_{4}=\left(0,0, x_{3}^{*}(0)\right)$, $M_{5}=\left(0, \bar{x}_{2}^{1}(0), \bar{x}_{3}^{1}(0)\right), M_{6}=\left(\bar{x}_{1}^{2}(0), 0, \bar{x}_{3}^{2}\right)$ and $M_{7}=\left(\bar{x}_{1}^{3}(0), \bar{x}_{2}^{3}, 0\right)$. Then, all $M_{i}(1 \leq i \leq 7)$ are fixed points of $Q$. For any $x \in X$, let $\omega(x)$ be the $\omega$-limit set of $x$ with respect to the discrete semiflow $\left\{Q^{n}\right\}_{n=0}^{\infty}$. Then, by our previous analysis, $\underset{x \in \partial X_{0}}{\cup} \omega(x)=\left\{M_{1}, M_{2}, \ldots, M_{7}\right\}$, and no subset of the $M_{i}$ 's forms a cycle for $\left.Q\right|_{\partial X_{0}}: \partial X_{0} \rightarrow \partial X_{0}$. By conditions (1), (2) and (3), and Lemma 1 in the Appendix, each $M_{i}(1 \leq i \leq 7)$ is isolated for $Q$ in $X_{0}$, and hence isolated in $X$, since $M_{i}$ is isolated for $\left.Q\right|_{\partial X_{0}}$ in $\partial X_{0}$, and $Q: X_{0} \rightarrow X_{0}$. Therefore, $\cup_{i=1}^{7} M_{i}$ is an isolated and acyclic covering of $\underset{x \in \partial X_{0}}{\cup} \omega(x)$ in $\partial X_{0}$. Again by Lemma 1 in the Appendix, for each $1 \leq i \leq 7, W^{s}\left(M_{i}\right) \cap X_{0}=\emptyset$. By [20, Theorem 2.2], it follows that $Q: X \rightarrow X$ is uniformly persistent with respect to $\left(X_{0}, \partial X_{0}\right)$. Therefore, by [20, Theorem 2.3], $Q$ has a global attractor $A_{0} \subset X_{0}$ relative to strongly bounded sets in $X_{0}$, and hence $A_{0}$ is globally asymptotically stable for $Q$ in $X_{0}$.

Let $(S(t), x(t))=\left(S(t), x_{1}(t), x_{2}(t), x_{3}(t)\right)$ be any given solution of (4.1) with $S(0) \geq 0$ and $x_{i}(0)>0(1 \leq i \leq 3)$, and let

$$
V(t)=S(t)+\sum_{i=1}^{3} x_{i}(t), \quad t \geq 0
$$

Then, $S(t)>0, x_{i}(t)>0(1 \leq i \leq 3), t>0$, and $V(t)$ satisfies

$$
\frac{d V(t)}{d t}=\left(S^{0}(t)-V(t)\right) D_{0}(t)
$$


and so $(S(t), x(t))$ exists globally on $[0, \infty)$. Therefore, $\lim _{t \rightarrow \infty} \mid V(t)-$ $V_{0}^{*}(t) \mid=0$, and $x(t)$ satisfies the 3 -dimensional nonautonomous system

$$
\frac{d x_{i}}{d t}=x_{i}\left(\hat{P}_{i}\left(t, V(t)-\sum_{j=1}^{3} x_{j}\right)-D_{0}(t)\right), \quad 1 \leq i \leq 3,
$$

with

$$
\lim _{t \rightarrow \infty}\left(\hat{P}_{i}\left(t, V(t)-\sum_{i=1}^{3} x_{j}\right)-\hat{P}\left(t, V_{0}^{*}(t)-\sum_{i=1}^{3} x_{j}\right)\right)=0 \quad(1 \leq i \leq 3)
$$

uniformly for $x=\left(x_{1}, x_{2}, x_{3}\right)$ in any bounded subset of $R_{+}^{3}$. By the boundedness of $V(t)$, it easily follows that solutions of (4.6) are uniformly bounded in $R_{+}^{3}$. Let $\phi(t, s, x)(t \geq s)$ be the unique solution of (4.6) with $\phi(s, s, x)=$ $x \in R_{+}^{3}=X$. By [21, Proposition 3.2], $\phi(t, s, x), t \geq s, x \in R_{+}^{3}$ is asymptotic to the $\omega$-periodic semiflow $T(t)=\phi_{0}(t, \cdot): R_{+}^{3} \rightarrow R_{+}^{3}$, and hence $T_{n}(x)=\phi(n \omega, 0, x): X \rightarrow X, n \geq 0$ is an asymptotically autonomous discrete dynamical process with limiting autonomous discrete semiflow $Q^{n}$ : $X \rightarrow X, n \geq 0$, where $Q=T(\omega)$ is the Poincaré map associated with (4.4) (see [21]). By conditions (1), (2) and (3) and Lemma 2 in the Appendix, for each $1 \leq i \leq 7, \widetilde{W}^{s}\left(M_{i}\right) \cap X_{0}=\emptyset$. Therefore, by [21, Theorem 2.5], for any $x \in X_{0}$, the $\omega$-limit set $\omega(x)$ of $\gamma^{+}(x)=\left\{T_{n}(x): n \geq 0\right\}$ satisfies $\omega(x) \subset A_{0}$. Then, by [21, Theorem 3.1], for any $x \in X_{0}$,

$$
\lim _{t \rightarrow \infty} d\left(\phi(t, 0, x), T(t) A_{0}\right)=0,
$$

and so, since $T(\omega) A_{0}=A_{0}$ and $T(t)$ is an $\omega$-periodic semiflow,

$$
\lim _{t \rightarrow \infty} d\left(\phi(t, 0, x), A_{0}^{*}\right)=0,
$$

where $A_{0}^{*}=\bigcup_{t \in[0, \omega]} T(t) A_{0}$ is a compact subset of $X_{0}$. In particular, since $x(0) \in X_{0}$,

$$
\lim _{t \rightarrow \infty} d\left(x(t), A_{0}^{*}\right)=\lim _{t \rightarrow \infty} d\left(\phi(t, 0, x(0)), A_{0}^{*}\right)=0 .
$$

Therefore, there exist $\alpha>0$ and $\beta>0$, which depend only on $A_{0}^{*}$, such that the solution $(S(t), x(t))$ of $(4.1)$ with $S(0) \geq 0$ and $x_{i}(0)>0(1 \leq i \leq 3)$ satisfies

$$
0<\alpha \leq \liminf _{t \rightarrow \infty} x_{i}(t) \leq \limsup _{t \rightarrow \infty} x_{i}(t) \leq \beta, \quad 1 \leq i \leq 3 .
$$

From the last part of the proof of Theorem 3.1, it follows that system (4.1) admits a positive periodic solution. This completes the proof. 
Theorem 4.2. Let (A2) hold. Assume that

(1) $\mu_{i} \equiv \int_{0}^{\omega}\left(P_{i}\left(t, V_{0}^{*}(t)\right)-D_{0}(t)\right) d t>0, \quad 1 \leq i \leq 3$;

(2) $\mu_{j i} \equiv \int_{0}^{\omega}\left(P_{i}\left(t, V_{0}^{*}(t)-x_{j}^{*}(t)\right)-D_{0}(t)\right) d t>0, \quad 1 \leq i, j \leq 3, i \neq$ $j, j \neq 2$, and $\mu_{21} \equiv \int_{0}^{\omega}\left(P_{1}\left(t, V_{0}^{*}(t)-x_{2}^{*}(t)\right)-D_{0}(t)\right) d t>0$,

(3) $\bar{\mu}^{i} \equiv \int_{0}^{\omega}\left(P_{i}\left(t, V_{0}^{*}(t)-\sum_{j=1, j \neq i}^{3} \bar{x}_{j}^{i}(t)\right)-D_{0}(t)\right) d t>0, \quad 2 \leq i \leq 3$, where $x_{i}^{*}(t)(1 \leq i \leq 3),\left(\bar{x}_{1}^{2}(t), \bar{x}_{3}^{2}(t)\right)$ and $\left(\bar{x}_{1}^{3}(t), \bar{x}_{2}^{3}(t)\right)$ are as in Theorem 4.1.

Then the conclusion of Theorem 4.1 holds.

Proof. We use the same notation as in the proof of Theorem 4.1. By the a priori estimate on the positive periodic solution of $\left(\hat{E}_{1}\right)$ claimed in the proof of Theorem 4.1, (A2) implies that $\left(\hat{E}_{1}\right)$ admits no positive periodic solution. Since $\int_{0}^{\omega}\left(P_{2}\left(t, V_{0}^{*}(t)-x_{3}^{*}(t)\right)-D_{0}(t)\right) d t>0$, by an argument using the theory of monotone dynamical systems, as in the proof of [6, Theorem 34.1], it follows that $\left(u_{2}^{*}(t), 0\right)$ is globally attractive for $\left(\hat{E}_{1}\right)$ in $\operatorname{int}\left(R_{+}^{2}\right)$. Clearly, $\bigcup_{x \in \partial X_{0}} \omega(x)=\left\{M_{1}, M_{2}, M_{3}, M_{4}, M_{6}, M_{7}\right\}$. Then, as in the proof of Theorem 4.1, $\cup_{i=1, i \neq 5}^{7} M_{i}$ is an isolated and acyclic covering of $\underset{x \in \partial X_{0}}{\cup} \omega(x)$ in $\partial X_{0}$. Now an argument similar to that given in Theorem 4.1 completes the proof.

Theorem 4.3. Let (A3) hold. Assume that

(1) $\mu_{i} \equiv \int_{0}^{\omega}\left(P_{i}\left(t, V_{0}^{*}(t)\right)-D_{0}(t)\right) d t>0, \quad 1 \leq i \leq 3$

(2) $\mu_{3 i} \equiv \int_{0}^{\omega}\left(P_{i}\left(t, V_{0}^{*}(t)-x_{3}^{*}(t)\right)-D_{0}(t)\right) d t>0, \quad 1 \leq i \leq 2, \mu_{21} \equiv$ $\int_{0}^{\omega}\left(P_{1}\left(t, V_{0}^{*}(t)-x_{2}^{*}(t)\right)-D_{0}(t)\right) d t>0$, and $\mu_{13} \equiv \int_{0}^{\omega}\left(P_{3}\left(t, V_{0}^{*}(t)-\right.\right.$ $\left.\left.x_{1}^{*}(t)\right)-D_{0}(t)\right) d t>0$

(3) $\bar{\mu}^{2} \equiv \int_{0}^{\omega}\left(P_{2}\left(t, V_{0}^{*}(t)-\sum_{j=1, j \neq 2}^{3} \bar{x}_{j}^{2}(t)\right)-D_{0}(t)\right) d t>0$, where $x_{i}^{*}(t)$ $(1 \leq i \leq 3)$ and $\left(\bar{x}_{1}^{2}(t), \bar{x}_{3}^{2}(t)\right)$ are as in Theorem 4.1 .

Then the conclusion of Theorem 4.1 holds.

Proof. Again we use the same notation as in the proof of Theorem 4.1.

As in the proof of Theorem 4.2, (A3) implies that $\left(u_{2}^{*}(t), 0\right)$ and $\left(u_{1}^{*}(t), 0\right)$ are globally attractive for $\left(\hat{E}_{1}\right)$ and $\left(\hat{E}_{3}\right)$ in $\operatorname{int}\left(R_{+}^{2}\right)$, respectively. Clearly, $\bigcup_{x \in \partial X_{0}} \omega(x)=\left\{M_{1}, M_{2}, M_{3}, M_{4}, M_{6}\right\}$. Then, as in the proof of Theorem 4.1, $\cup_{i=1, i \neq 5}^{6} M_{i}$ is an isolated and acyclic covering of $\underset{x \in \partial X_{0}}{\cup} \omega(x)$ in $\partial X_{0}$. Now again, an argument similar to that given in Theorem 4.1 completes the proof.

Remark 4.4. If, instead of assumption (A1), we let $M_{5}, M_{6}$ and $M_{7}$, in the proof of Theorem 4.1, be three positive, global attractors of the Poincaré maps associated with the three, 2-dimensional competition systems $\left(\hat{E}_{1}\right),\left(\hat{E}_{2}\right)$ and $\left(\hat{E}_{3}\right)$, respectively, then, by a similar argument, the conclusion of Theorem 4.1 holds with condition (3) replaced by a revised 

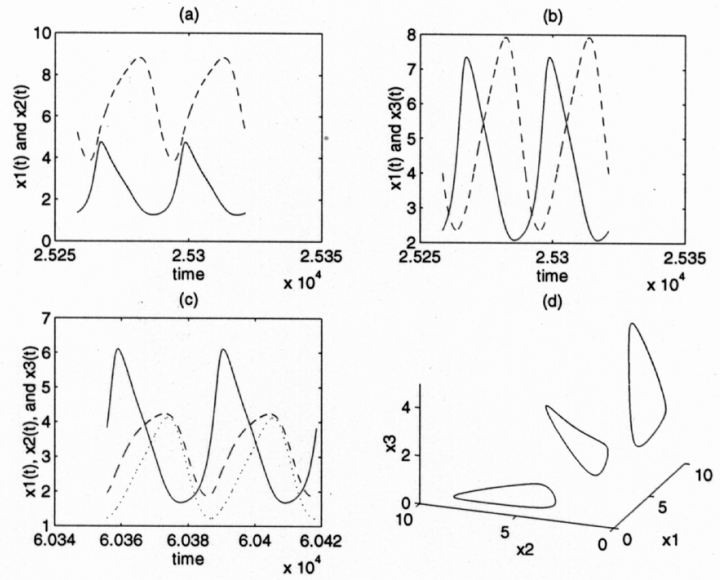

Figure 1. An example of competition-mediated oscillatory coexistence of three species illustrating Theorem 4.2. Figure 1(a) depicts the time series of the globally attracting $\omega$-periodic solution in the $x_{1}-x_{2}$ face. The solid curve is $x_{1}(t)$ and the dashed curve is $x_{2}(t)$. Figure $1(\mathrm{~b})$ depicts the time series of the globally attracting $\omega$-periodic solution in the $x_{1}-x_{3}$ face. The solid curve is $x_{1}(t)$ and the dashed curve is $x_{3}(t)$. Figure 1(c) depicts the time series of the positive $\omega$-periodic solution. Figure 1(d) gives the projection into $x_{1}-x_{2}-x_{3}$ phase-space, of the twoand three-species $\omega$-periodic orbits shown in Figure 1(a)-(c).

invasibility condition. For example, let $\left(\underline{x}_{1}(t), \underline{x}_{2}(t)\right)$ and $\left(\bar{x}_{1}(t), \bar{x}_{2}(t)\right)$ be as in the proof of Theorem 4.1. Then, under condition (3), with $i=3$ and $\left(\bar{x}_{1}^{3}(t), \bar{x}_{2}^{3}(t)\right)$ replaced by $\left(\bar{x}_{1}(t), \underline{x}_{2}(t)\right)$, one can prove that $M_{7}$ is an isolated invariant set of $Q$ in $X$ and $\widetilde{W}^{s}\left(M_{7}\right) \cap X_{0}=\emptyset$, by using the compressivity of $\left(\hat{E}_{3}\right)$ and arguments similar to those given in Lemmas 1 and 2 in the Appendix.

\subsection{An example of oscillatory competition-mediated coexis-} tence. In this section an example is given to illustrate the oscillatory coexistence of three species predicted by Theorem 4.2.

All of the simulations are done using MATLAB. We assumed convergence occurred when $x_{i}(t)$ and $x_{i}(t+\omega)$ agreed to at least 10 decimal places.

Example. We use the parameters from the three-species example given in Lenas and Pavlou [9, Table 1, case (a) and Figure 3(a)]. The operating parameters $u_{0}=0.4675$ and $S^{0}(t) \equiv 11$ were selected so that the dynamics would be given by region 27 of their bifurcation diagram (see [9, Figure 
3(a) and Table 3]). The dilution rate was therefore given by $D_{0}(t)=\mu_{0}+$ $a \cos (\omega t)$, where $a=0.3$ and $\omega=0.2$ and Monod-type functional responses (see $\left[9\right.$, Table 1, case (a)]) $P_{i}(t, S(t))=\alpha_{i} S(t) /\left(\beta_{i}+S(t)\right)$, where $\alpha_{1}=$ $1, \beta_{1}=1, \alpha_{2}=0.7, \beta_{2}=0.3, \alpha_{3}=0.64$, and $\beta_{3}=0.2$.

From the simulations it appears that all of the hypotheses of Theorem 4.2 are satisfied. See Figure 1, where we plot the time series of the unique two-species positive periodic solution in the $x_{1}-x_{2}$ face in Figure 1 (a) and the unique two-species positive periodic solution in the $x_{1}-x_{3}$ face in Figure 1(b). According to [9, Table 3] (and our simulations confirm this), for the parameters selected, there is no positive periodic solution in the $x_{2}-x_{3}$ face. Instead all orbits in this face approach the single-species $\omega$-periodic solution where $x_{2}(t)>0$ but $x_{3}(t) \equiv 0$.

We evaluated the integrals given in Theorem 4.2 numerically using the trapezoidal rule as in the previous example and found $\mu_{1}=14.1111, \mu_{2}=$ $6.7205, \mu_{3}=5.0603, \mu_{12}=0.6780, \mu_{13}=1.87861, \mu_{21}=0.7909, \mu_{31}=$ $0.2990, \mu_{32}=0.8122, \bar{\mu}^{2}=0.0012$, and $\bar{\mu}^{3}=0.0009$. Note that all of these quantities are positive and that it is not necessary to evaluate $\mu_{23}$ in this case.

In Figure 1(c), the time series of the three-species positive $\omega$-periodic solution, predicted to exist by Theorem 4.2 , is shown.

This example also illustrates competition-mediated coexistence in the following sense. If species one is absent, and species two and three compete, then species two drives species three to extinction. However, this extinction of species three is avoided simply by introducing competitor one. Once competitor one is introduced, all three species persist in sustained oscillation.

Appendix. In this appendix, we give two results that are very useful in the application of the abstract uniform persistence (or repellor) theorems for the periodic and asymptotically periodic semiflows to the m-dimensional Kolmogorov periodic and asymptotically periodic biological systems.

Consider first the m-dimensional Kolmogorov periodic system

$$
\frac{d u_{i}}{d t}=u_{i} F_{0 i}(t, u) \quad(1 \leq i \leq m)
$$

where $u=\left(u_{1}, \ldots, u_{m}\right) \in R_{+}^{m}$. We assume that $F_{0}=\left(F_{01}, \ldots, F_{0 m}\right)^{T}$ : $R_{+}^{m+1} \rightarrow R_{+}^{m}$ is continuous and $\omega$-periodic with respect to $t(\omega>0)$, and that the solution $\phi_{0}(t, u)$ of (A1) with $\phi_{0}(0, u)=u$ exists uniquely on $[0, \infty)$. Let $S=\phi_{0}(\omega, \cdot): R_{+}^{n} \rightarrow R_{+}^{m}$. Then, $S^{n}(u)=\phi_{0}(n \omega, u)$ for any $u \in R_{+}^{m}$.

Lemma 1. If for some $1 \leq i \leq m$,

$$
u^{*}(t)=\left(u_{1}^{*}(t), \ldots, u_{i-1}^{*}(t), 0, u_{i+1}^{*}(t), \ldots, u_{m}^{*}(t)\right)
$$


is an $\omega$-periodic solution of (A1) with $u_{j}^{*}(0) \geq 0(1 \leq j \leq m, j \neq i)$ and $u^{*}(t)$ satisfies $\int_{0}^{\omega} F_{0 i}\left(t, u^{*}(t)\right) d t>0$. Then, there exists $\delta>0$ such that

$$
\varlimsup_{n \rightarrow \infty} d\left(S^{n}(u), u^{*}(0)\right) \geq \delta, \text { for all } u \in \operatorname{int}\left(R_{+}^{m}\right) .
$$

Proof. It suffices to prove that there exists $\delta>0$ such that for any $u \in$ $B\left(u^{*}(0), \delta\right) \cap \operatorname{int}\left(R_{+}^{m}\right)$, where $B\left(u^{*}(0), \delta\right)=\left\{u \in R^{m}:\left|u-u^{*}(0)\right|<\delta\right\}$, there exists $N=N(u) \geq 1$ such that $S^{N}(u) \notin B\left(u^{*}(0), \delta\right)$. Let $\epsilon$ be a fixed positive number such that $0<\epsilon<\frac{1}{\omega} \int_{0}^{\omega} F_{0 i}\left(t, u^{*}(t)\right) d t$. By the uniform continuity of $F_{0 i}(t, u)$ on the compact subset $[0, \omega] \times[0, b]^{m} \subset R^{m+1}$, where $b=\max _{0 \leq t \leq \omega}\left|u^{*}(t)\right|+1$, there exists $\delta_{0} \in(0,1)$ such that for any $u$ and $v \in[0, b]^{m}$ with $|u-v|<\delta_{0}$, and all $t \in[0, \omega]$,

$$
\left|F_{0 i}(t, u)-F_{0 i}(t, v)\right|<\epsilon
$$

By the continuous dependence of solutions on initial values, it then follows that there exists $\delta>0$ such that for any $u \in B\left(u^{*}(0), \delta\right) \cap R_{+}^{m}$,

$$
\left|\phi_{0}(t, u)-u^{*}(t)\right|=\left|\phi_{0}(t, u)-\phi_{0}\left(t, u^{*}(0)\right)\right|<\delta_{0}, \quad \text { for all } t \in[0, \omega] .
$$

Proceeding by contradiction, assume that there exists $u_{0} \in B\left(u^{*}(0), \delta\right) \cap$ $\operatorname{int}\left(R_{+}^{m}\right)$ such that for all $n \geq 1, S^{n}\left(u_{0}\right)=\phi_{0}\left(n \omega, u_{0}\right) \in B\left(u^{*}(0), \delta\right)$. For any $t \geq 0$, let $t=n \omega+t^{\prime}$, where $t^{\prime} \in[0, \omega)$ and $n=[t / \omega]$ is the greatest integer less than or equal to $t / \omega$. Then,

$$
\left|\phi_{0}\left(t, u_{0}\right)-u^{*}(t)\right|=\left|\phi_{0}\left(t^{\prime}, \phi_{0}\left(n \omega, u_{0}\right)\right)-u^{*}\left(t^{\prime}\right)\right|<\delta_{0} .
$$

Therefore,

$$
\left|F_{0 i}\left(t, \phi_{0}\left(t, u_{0}\right)\right)-F_{0 i}\left(t, u^{*}(t)\right)\right|<\epsilon, \quad \text { for all } t \geq 0 .
$$

Let $\phi_{0}\left(t, u_{0}\right)=\left(\phi_{1}\left(t, u_{0}\right), \ldots, \phi_{m}\left(t, u_{0}\right)\right)$. Then, for all $t \geq 0, \phi_{0}\left(t, u_{0}\right) \in$ $\operatorname{int}\left(R_{+}^{m}\right)$, and hence $\phi_{i}\left(t, u_{0}\right)$ satisfies

$$
\frac{d \phi_{i}}{d t}=\phi_{i} F_{0 i}\left(t, \phi_{0}(t)\right) \geq \phi_{i}\left(F_{0 i}\left(t, u^{*}(t)\right)-\epsilon\right), \quad t \geq 0 .
$$

Therefore, by the comparison theorem,

$$
\phi_{i}\left(t, u_{0}\right) \geq \phi_{i}\left(0, u_{0}\right) \cdot e^{\int_{0}^{t}\left(F_{0 i}\left(t, u^{*}(t)\right)-\epsilon\right) d s}, \text { for all } t \geq 0 .
$$

In particular,

$$
\phi_{i}\left(n \omega, u_{0}\right) \geq \phi_{i}\left(0, u_{0}\right) \cdot e^{n \int_{0}^{\omega}\left(F_{0 i}\left(t, u^{*}(t)\right)-\epsilon\right) d s}, \text { for all } n \geq 0 .
$$


By the choice of $\epsilon, \lim _{n \rightarrow \infty} \phi_{i}\left(n \omega, u_{0}\right)=+\infty$, which contradicts our assumption that $S^{n}\left(u_{0}\right)=\phi_{0}\left(n \omega, u_{0}\right) \in B\left(u^{*}(0), \delta\right)$ for all $n \geq 1$. Therefore, for all $u \in \operatorname{int}\left(R_{+}^{m}\right), \varlimsup_{n \rightarrow \infty} d\left(S^{n}(u), u^{*}(0)\right) \geq \delta$. This completes the proof.

Then, consider the m-dimensional nonautonomous Kolmogorov system

$$
\frac{d u_{i}}{d t}=u_{i} F_{i}(t, u) \quad(1 \leq i \leq m)
$$

where $u=\left(u_{1}, \ldots, u_{m}\right) \in R_{+}^{m}$. We assume that $F=\left(F_{1}, \ldots, F_{m}\right)^{T}$ : $R_{+}^{m+1} \rightarrow R_{+}^{m}$ is continuous and locally Lipschitz in $u$. Let $\phi_{0}(t, s, u)$ and $\phi(t, s, u)$ be the unique solutions of (A1) and (A2) with $\phi_{0}(s, s, u)=u$ and $\phi(s, s, u)=u(s \geq 0)$, respectively. Let $T_{n}(u)=\phi(n \omega, 0, u), T(t) u=$ $\phi_{0}(t, 0, u)$ and $S(u)=T(\omega) u, u \in R_{+}^{m}$.

Lemma 2. Assume that $\lim _{t \rightarrow \infty}\left|F(u, t)-F_{0}(t, u)\right|=0$ uniformly for $u$ in any bounded subset of $R_{+}^{m}$, and that solutions of (A1) and (A2) are uniformly bounded in $R_{+}^{m}$. If for some $1 \leq i \leq m$,

$$
u^{*}(t)=\left(u_{1}^{*}(t), \ldots, u_{i-1}^{*}(t), 0, u_{i+1}^{*}(t), \ldots, u_{m}^{*}(t)\right)
$$

is an $\omega$-periodic solution of $(\mathrm{A} 1)$ with $u_{j}^{*}(0) \geq 0(1 \leq j \leq m, j \neq i)$ and $u^{*}(t)$ satisfies

$$
\int_{0}^{\omega} F_{0 i}\left(t, u^{*}(t)\right) d t>0
$$

then $\widetilde{W^{s}}\left(u^{*}(0)\right) \cap \operatorname{int}\left(R_{+}^{m}\right)=\emptyset$, where

$$
\widetilde{W}^{s}\left(u^{*}(0)\right)=\left\{u \in R_{+}^{m}: \lim _{n \rightarrow \infty} T_{n}(u)=u^{*}(0)\right\} .
$$

Proof. By [21, Proposition 3.2], $\phi(t, s, u), t \geq s \geq 0, u \in R_{+}^{m}$, is asymptotic to the $\omega$-periodic semiflow $T(t): R^{m} \rightarrow R^{m}$, and hence $T_{n}(u): R_{+}^{m} \rightarrow$ $R_{+}^{m}, n \geq 0$ is an asymptotically autonomous discrete dynamical process with the limiting autonomous discrete semiflow $S^{n}: R_{+}^{m} \rightarrow R_{+}^{m}, n \geq 0$ ([21]). Assume that, for the sake of contradiction, there exists a $u_{0} \in \widetilde{W}^{s}\left(u^{*}(0)\right) \cap$ $\operatorname{int}\left(R_{+}^{m}\right)$. Then, $\lim _{n \rightarrow \infty} T_{n}\left(u_{0}\right)=u^{*}(0)$, and hence, by [21, Theorem 3.1],

$$
\lim _{t \rightarrow \infty}\left(\phi\left(t, 0, u_{0}\right)-u^{*}(t)\right)=\lim _{t \rightarrow \infty}\left(\phi\left(t, 0, u_{0}\right)-T(t) u^{*}(0)\right)=0 .
$$

Since $\lim _{t \rightarrow \infty}\left|F(t, u)-F_{0}(t, u)\right|=0$ uniformly for $u$ in any bounded subset of $R_{+}^{m}$, it easily follows that

$$
\lim _{t \rightarrow \infty}\left(F\left(t, \phi\left(t, 0, u_{0}\right)\right)-F_{0}\left(t, u^{*}(t)\right)\right)=0 .
$$


In particular,

$$
\lim _{t \rightarrow \infty}\left(F_{i}\left(t, \phi\left(t, 0, u_{0}\right)\right)-F_{0 i}\left(t, u^{*}(t)\right)\right)=0 .
$$

Let $\epsilon$ be a fixed positive number such that $0<\epsilon<\frac{1}{\omega} \int_{0}^{\omega} F_{0 i}\left(t, u^{*}(t)\right) d t$. Then, there exists $N=N(\epsilon)>0$ such that for all $t \geq N \omega$,

$$
F_{i}\left(t, \phi\left(t, 0, u_{0}\right)\right) \geq F_{0 i}\left(t, u^{*}(t)\right)-\epsilon .
$$

Let $\phi\left(t, 0, u_{0}\right)=\left(\phi_{1}(t), \ldots, \phi_{n}(t)\right)=\phi(t)$. Then, $\phi(t) \in \operatorname{int}\left(R_{+}^{m}\right)$ for all $t \geq 0$. Therefore, $\phi_{i}(t)$ satisfies

$$
\frac{d \phi_{i}(t)}{d t}=\phi_{i}(t) F_{i}(t, \phi(t)) \geq \phi_{i}(t)\left(F_{0 i}\left(t, u^{*}(t)\right)-\epsilon\right), \quad t \geq N \omega .
$$

By the comparison theorem,

$$
\phi_{i}(t) \geq \phi_{i}(N \omega) e^{\int_{N \omega}^{t}\left(F_{0 i}\left(s, u^{*}(s)\right)-\epsilon\right) d s}, \quad t \geq N \omega .
$$

In particular, for all $n \geq N$,

$$
\phi_{i}(n \omega) \geq \phi_{i}(N \omega) e^{(n-N) \int_{0}^{\omega}\left(F_{0 i}\left(s, u^{*}(s)\right)-\epsilon\right) d s} .
$$

Then, by the choice of $\epsilon$,

$$
\lim _{n \rightarrow \infty} \phi_{i}(n \omega)=+\infty
$$

which contradicts

$$
\lim _{t \rightarrow \infty}\left(\phi\left(t, 0, u_{0}\right)-u^{*}(t)\right)=0 .
$$

This completes the proof.

\section{REFERENCES}

[1] G.J. Butler, S.B. Hsu, and P. Waltman, A mathematical model of the chemostat with periodic washout rate, SIAM J. Appl. Math., 45 (1985), 435-449.

[2] G.J. Butler and G.S.K. Wolkowicz, A mathematical model of the Chemostat with a general class of functions describing nutrient uptake, SIAM J. Appl. Math., 45 (1985), 138-151.

[3] W.A. Coppel, "Stability and Asymptotic Behaviour of Differential Equations," D. C. Heath, New York, 1965.

[4] P. deMottoni and A. Schiaffino, Competition systems with periodic coefficients: A geometric approach, J. Math. Biology, 11 (1982), 319-335.

[5] J.K. Hale and A.S. Somolinas, Competition for fluctuating nutrient, J. Math. Biology, 18 (1983), 255-280. 
[6] P. Hess, "Periodic-Parabolic Boundary Value Problems and Positivity," Pitman Research Notes in Math., Series 247, Longman Scientific and Technical, 1991.

[7] S.B. Hsu, A competition model for a seasonally fluctuating nutrient, J. Math. Biology, 9 (1980), 115-132.

[8] P. Lenas and S. Pavlou, Periodic, quasi-periodic and chaotic coexistence of two competing microbial populations in a periodically operated chemostat, Math. Biosci., 121 (1994), 61-110.

[9] P. Lenas and S. Pavlou, Coexistence of three competing microbial populations in a chemostat with periodically varying dilution rate, Math. Biosci., 129 (1995), 111142.

[10] H.L. Smith, Competitive coexistence in an oscillating chemostat, SIAM J. Appl. Math., 40 (1981), 498-522.

[11] H.L. Smith, Microbial growth in periodic gradostats, Rocky Mountain J. Math., 20 (1990), 1173-1194.

[12] H.L. Smith, Periodic rotating waves in a model of microbial competition in a circular gradostat, Canad. Appl. Math. Quart., 1 (1993), 83-113.

[13] H.L. Smith and P. Waltman, "The Theory of the Chemostat," Cambridge University Press, 1995.

[14] G. Stephanopoulos, A.G. Fredrickson, and R. Aris, The growth of competing microbial populations in CSTR with periodically varying inputs, American Institute of Chem. Eng. Journal, 25 (1979), 863-872.

[15] G.S.K. Wolkowicz and Z. Lu, Global dynamics of a mathematical model of competition in the chemostat: general response functions and differential death rates, SIAM J. Appl. Math., 52 (1992), 222-233.

[16] G.S.K. Wolkowicz and H. Xia, Global asymptotic behavior of a chemostat model with discrete delays, SIAM J. Appl. Math., 57 (1997), 1019-1043.

[17] F. Yang and H.I. Freedman, Competing predators for a prey in a chemostat model with periodic nutrient input, J. Math. Biol., 29 (1991), 715-732.

[18] T. Yoshizawa, "Stability Theory and the Existence of Periodic Solutions and Almost Periodic Solutions," Appl. Math. Sciences 14, Springer-Verlag, New York, 1975.

[19] X.-Q. Zhao, Global attractivity and stability in some monotone discrete dynamical systems, Bulletin of the Australia Mathematical Society, 53 (1996), 305-324.

[20] X.-Q. Zhao, Uniform persistence and periodic coexistence states in infinite-dimensional periodic semiflows with applications, Canad. Appl. Math. Quart., 3 (1995), 473-495.

[21] X.-Q. Zhao, Asymptotic behavior for asymptotically periodic semiflows with applications, Communications on Applied Nonlinear Analysis, 3 (1996), 43-66.

[22] X.-Q. Zhao and V. Hutson, Permanence in Kolmogorov periodic predator-prey models with diffusion, Nonlinear Analysis, 23 (1994), 651-668. 